\title{
Clinicopathological Features, Clinical Efficacy on 101 Cases of Rectal Gastrointestinal Stromal Tumors, and the Significance of Neoadjuvant Therapy
}

\author{
Hongxin Yang \\ Sichuan University \\ Chaoyong Shen \\ Sichuan University \\ Xiaonan Yin \\ Sichuan University \\ Zhaolun Cai \\ Sichuan University \\ Qian Wang \\ Affiliated Hospital of Guizhou Medical University \\ Bo Zhang ( $\sim$ hxwcwk@126.com ) \\ Sichuan University
}

\section{Research Article}

Keywords: Rectal, Gastrointestinal stromal tumor, Neoadjuvant treatment, Imatinib Mesylate, Disease-free survival, Overall survival

Posted Date: September 22nd, 2021

DOI: https://doi.org/10.21203/rs.3.rs-829937/v1

License: (c) (i) This work is licensed under a Creative Commons Attribution 4.0 International License. Read Full License

Version of Record: A version of this preprint was published at BMC Surgery on November 19th, 2021. See the published version at https://doi.org/10.1186/s12893-021-01397-8. 


\section{Abstract}

Objective: To investigate the clinicopathological features, clinical efficacy on 101 cases of rectal gastrointestinal stromal tumors (GISTs), and the significance of Imatinib Mesylate (IM) neoadjuvant therapy.

Methods: The clinicopathological features, treatment methods, peri-operative data, and prognosis of the patients were summarized and analyzed on 101 patients with rectal GISTs, who received treatment in the Gastrointestinal Department of West China Hospital of SichuanUniversity and the Affiliated Hospital of Guizhou Medical University from August 2002 toNovember 2020 in China.

Results: A total of 101 patients, including 64 males and 37 females, were aged from 22 to 79 years (55.4 112.2 years). Among 70 patients with direct surgery, which included 8 very low risk cases, 10 low risk cases, 7 intermediate risk cases, and 45 high risk cases. Cox regression analysis showed that post-operative IM adjuvant treatment improved disease-free survival (DFS) and overall survival (OS) of 52 intermediate and high risk patients. Among the 31 patients who received neoadjuvant therapy, the objective response rate (ORR) was $83.9 \%$ (26/31), and the disease control rate (DCR) reached 96.8\% (30/31). Diameter subgroup analysis: (1) Among the 36 patients with a diameter $\leq 5 \mathrm{~cm}$, two patients received IM neoadjuvant therapy, while 34 patients received direct surgery. Both univariate and Cox regression analysis did not find that neoadjuvant therapy affects DFS and OS. (2) Among the 65 patients of diameter $>5 \mathrm{~cm}, 29$ received IM neoadjuvant therapy and 36 received direct surgery. Patients who underwent neoadjuvant therapy had less blood loss $(P=0.022)$, shorter post-operative hospital stay $(P=0.001)$, increased anal preservation proportion (93.1\% VS72.2\%, P=0.031), decreased enterostomy proportion (10.3\% VS 33.3\%, $\mathrm{P}=0.037$ ) than those who underwent direct surgery. Cox regression analysis suggested that neoadjuvant therapy and post-operative IM adjuvant therapy improved DFS.

Conclusion: Rectal GISTs is relatively rare and is a highly malignant tumor, post-operative oral IM therapy can improve DFS and OS of intermediate and high risk patients. In patients with rectal GISTs with diameter $>5 \mathrm{~cm}$, IM neoadjuvant therapy can improve the anal preservation proportion, preserve the structure and function of the organs, reduce enterostomy proportion, and improve prognosis.

\section{Introduction}

Rectal gastrointestinal stromal tumors (GISTs) account for 3-5\% of all GISTs, less than that of stomach and small intestine [1-3]. The onset of rectal GIST is rare and insidious, and it is anatomically located in the narrow pelvis, which is close to important structures such as the reproductive and urinary systems. In addition, the tumor is close to the dentate line, and the operation may damage the anal sphincter. These factors above make anal preservation challenging $[4,5]$. Currently, the clinicopathological features and treatment methods for rectal GISTs, especially large sample size of IM neoadjuvant therapy of rectal GIST patients has seldomly been reported domestic and abroad. In the present study, data were obtained from 101 patients with rectal GISTs who received treatment in the West ChinaHospital of Sichuan University and the Affiliated Hospital of Guizhou Medical University.This study may help in understanding the clinicopathological characteristics, prognosis of rectal GISTs, especially the significance of IM neoadjuvant therapy.

\section{Patients And Methods}

\section{Patient selection}


Hospitalization data were obtained, and follow-up was conducted on 101 patients with rectal GISTs who were treated in West China Hospital of Sichuan University and the Affiliated Hospital of Guizhou Medical University from August 2002 to November 2020. The study was approved by the Institutional Review Board of each institution, and all patients provided informed consent for participation. The inclusion criteria are as follows: (1) 18 years $\leq$ age $<80$ years; (2) Rectal GISTs was confirmed by post-operative pathology, immunohistochemical examination or genetic testing after surgical resection; (3) Distant metastasis was excluded by chest and abdominal CT examination; (4) Patients without serious heart, lung, kidney and other complications can tolerate targeted therapy; (5) The Eastern Cooperative Oncology Group (ECOG) Performance Score < 2; and ; (6) Patients with complete clinical data and followup. The exclusion criteria are as follows: (1) Pregnant or lactating women (age < 18 years); (2) Patients with other major systemic diseases or have a distant metastasis; (3) Patients whose clinical data were incomplete or lost to follow-up.

\section{Clinical case collection}

All data, including age, gender, patient history, clinical symptoms, imaging data, ECOG Performance Score, type of operation, surgical data (including operation time, blood loss, postoperative hospital stay, anus reservation proportion, enterostomy proportion), postoperative treatment and prognosis data, was collected retrospectively and analyzed. Resected specimens were reviewed by pathologists from each institution and the risk of recurrence after surgery was classified according to the modified National Institutes of Health (NIH) criteria [6].

\section{Therapeutic method}

Patients received direct surgery: After operation,patients were classified into very low, low, intermediate and high risk according to the modified $\mathrm{NIH}$ criteria. Periodic follow-up is needed for very low and low risk patients. IM was oral administered for at least 1 year for intermediate risk patients and at least 3 years for high risk patients after surgery.

Patients received IM neoadjuvant therapy: Patients were administered with $400 \mathrm{mg} / \mathrm{day}$ IM tablets preoperatively for at least 3 months. The patients needed check-up every 3 months. The evaluation was performed according to Choi criteria for the efficacy evaluation of modified solid tumors [7]. Those with favorable response after neoadjuvant therapy accepted surgery. A dose of $400-600 \mathrm{mg} /$ day IM was suggestted taking orally after surgery according to genetic result.

\section{Follow up}

A total of 101 patients were followed up until March 31, 2021, and the median follow-up time was 65.7 months (6-240 months). After surgery, patients entered regular outpatient follow-up using a combination of blood tests, rectal palpation, colonoscopy and imaging evaluation at determined intervals. Disease-free survival (DFS) was defined as the time from the date of surgery to the time of recurrence or death due to disease progression, while overall survival (OS) was defined as the time from the date of surgery to the last follow-up or death. Patients with intermediate and high risk were followed up every 3 months in the first 3 years, every 6 months in the following 2 years, and then annually thereafter. Very low or low risk patients were followed up every 6 months for the first 5 years and annually thereafter. Follow-up was stopped until the patient died.

\section{Statistical analysis}

SPSS 22.0 statistical software was used to analyze the data. Measurement data were expressed as mean \pm standard deviation $(x \pm s)$, and t test was used. Enumerative data were expressed as absolute numbers and percentages and were compared by $\chi 2$ test or Fisher's exact probability method. Kaplan-Meier was used to draw the survival curve 
(Log-rank was used for difference test). Cox survival regression was used for survival regression analysis, and $\mathrm{P}<$ 0.05 was considered statistically significant.

\section{Results}

A total of 3316 GIST patients,including 138 rectal GISTs, were enrolled in the two hospitals. Excluding 37 patients who were lost to follow-up, having distant metastasis or whose data were incomplete, 101 patients with rectal GISTs were included in this study, and the clinicopathological characteristics and treatment methods information are shown detailedly in Table 1. Of the 101 patients, 35 patients recurred, $33.9 \pm 21.1$ months (5-96 months), and 28 of them

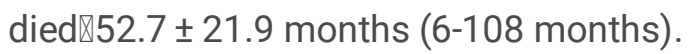


Table 1

The clinicopathological characteristics, surgical data of IM neoadjuvant therapy and direct surgery for all 101 rectal GIST patients

\begin{tabular}{|c|c|c|c|}
\hline Variables & $\begin{array}{l}\text { IM neoadjuvant therapy }(N= \\
\text { 31) }\end{array}$ & $\begin{array}{l}\text { Direct surgery }(N= \\
70)\end{array}$ & $\begin{array}{l}\text { Number }(N= \\
101) \\
(N / \%)\end{array}$ \\
\hline \multicolumn{4}{|l|}{ Age $^{a}$ (years) } \\
\hline$\leq 60$ & 17 & 45 & $62(61.4)$ \\
\hline$>60$ & 14 & 25 & $39(38.6)$ \\
\hline \multicolumn{4}{|l|}{ Gender } \\
\hline Male & 17 & 47 & $64(63.4)$ \\
\hline Female & 14 & 23 & $37(36.6)$ \\
\hline \multicolumn{4}{|l|}{ ECOG Performance Score } \\
\hline 0 & 29 & 62 & $91(90.1)$ \\
\hline 1 & 2 & 8 & $10(9.9)$ \\
\hline \multicolumn{4}{|l|}{ Intial clinical manifestation } \\
\hline Rectal bleeding & 10 & 10 & $20(19.8)$ \\
\hline Change in bowel of stool & 4 & 11 & $15(14.9)$ \\
\hline Change in bowel habit & 5 & 13 & $18(17.8)$ \\
\hline Rectal discomfort & 5 & 12 & $17(16.8)$ \\
\hline Digital rectal examination ${ }^{b}$ & 3 & 15 & $18(17.8)$ \\
\hline Others & 4 & 9 & $13(12.9)$ \\
\hline \multicolumn{4}{|l|}{ Tumor size (cm) } \\
\hline$\leq 5$ & 2 & 34 & $36(35.6)$ \\
\hline$>5$ & 29 & 36 & $65(64.4)$ \\
\hline \multicolumn{4}{|l|}{ Distance from anal verge } \\
\hline$\leq 7$ & 23 & 56 & $79(78.2)$ \\
\hline$>7$ & 8 & 14 & $22(21.8)$ \\
\hline \multicolumn{4}{|l|}{ Type of surgery } \\
\hline Laparotomy & 25 & 57 & $82(81.2)$ \\
\hline Laparoscopy & 6 & 8 & 14(13.9) \\
\hline
\end{tabular}

a Age:22-79 years (55.4 \pm 12.2 years); b $17.8 \%$ of the patients were found initally durning the rectal palpation, and a total of $69.3 \%(70 / 101)$ of all the patients could be found by this examination; $c$ Only 78 patients underwent DOG-1 testing; d Excluding 31 neoadjuvant patients who could not applicable to modified NIH criteria,70 patients left; e Only 47 patients underwent genetic testing. 


\begin{tabular}{|c|c|c|c|}
\hline Variables & $\begin{array}{l}\text { IM neoadjuvant therapy }(N= \\
\text { 31) }\end{array}$ & $\begin{array}{l}\text { Direct surgery ( } N= \\
\text { 70) }\end{array}$ & $\begin{array}{l}\text { Number }(N= \\
101) \\
(N / \%)\end{array}$ \\
\hline Endoscopy & 0 & 5 & $5(4.9)$ \\
\hline \multicolumn{4}{|l|}{ Surgical scope } \\
\hline Local & 30 & 65 & 95(94.1) \\
\hline Radical & 1 & 5 & $6(5.9)$ \\
\hline \multicolumn{4}{|l|}{ Surgical margin } \\
\hline positive & 0 & 4 & $4(4.0)$ \\
\hline negative & 31 & 66 & $97(96.0)$ \\
\hline \multicolumn{4}{|l|}{ Tumor rupture } \\
\hline Yes & 0 & 0 & $0(0.0)$ \\
\hline No & 31 & 70 & $101(100.0)$ \\
\hline \multicolumn{4}{|c|}{ Mitotic index (50HPF) } \\
\hline$\leq 5$ & 22 & 28 & $50(49.5)$ \\
\hline$>5$ & 9 & 42 & $51(50.5)$ \\
\hline \multicolumn{4}{|c|}{ Pathological feature } \\
\hline Spindle & 28 & 54 & $82(81.2)$ \\
\hline Epithelial & 2 & 13 & $15(14.9)$ \\
\hline mixed & 1 & 3 & $4(3.9)$ \\
\hline \multicolumn{4}{|c|}{ Immunohistochemistry } \\
\hline CD117 positive & 29 & 69 & 98/101 (97.0) \\
\hline DOG-1positive ${ }^{\mathrm{C}}$ & 29 & 46 & 75/78 (96.2) \\
\hline CD34 positive & 28 & 55 & $83 / 101(82.1)$ \\
\hline \multicolumn{4}{|c|}{ Modified NIH criteriad } \\
\hline Very low risk & - & 8 & $8(11.4)$ \\
\hline Low risk & - & 10 & $10(14.3)$ \\
\hline Medium risk & - & 7 & $7(10.0)$ \\
\hline High risk & - & 45 & 45 (64.3) \\
\hline
\end{tabular}

a Age: $22-79$ years ( $55.4 \pm 12.2$ years); b $17.8 \%$ of the patients were found initally durning the rectal palpation, and a total of $69.3 \%(70 / 101)$ of all the patients could be found by this examination; $c$ Only 78 patients underwent DOG-1 testing; d Excluding 31 neoadjuvant patients who could not applicable to modified NIH criteria,70 patients left; e Only 47 patients underwent genetic testing. 


\begin{tabular}{|c|c|c|c|}
\hline Variables & $\begin{array}{l}\text { IM neoadjuvant therapy }(N= \\
31)\end{array}$ & $\begin{array}{l}\text { Direct surgery ( } N= \\
\text { 70) }\end{array}$ & $\begin{array}{l}\text { Number }(N= \\
101) \\
(N / \%)\end{array}$ \\
\hline KIT Exon 11 mutation & 14 & 25 & $39(83.0)$ \\
\hline KIT Exon 9 mutation & 2 & 2 & $4(8.5)$ \\
\hline Wild type mutation & 1 & 3 & $4(8.5)$ \\
\hline \multicolumn{4}{|c|}{$\begin{array}{l}\text { Post-operative IM Adjuvant } \\
\text { treatment }\end{array}$} \\
\hline Yes & 25 & 24 & $49(48.5)$ \\
\hline No & 6 & 46 & $52(51.5)$ \\
\hline \multicolumn{4}{|c|}{$\begin{array}{l}\text { a Age:22-79 years ( } 55.4 \pm 12.2 \text { years); } \mathbf{b} 17.8 \% \text { of the patients were found initally durning the rectal palpation, and } \\
\text { a total of } 69.3 \%(70 / 101) \text { of all the patients could be found by this examination; c Only } 78 \text { patients underwent } \\
\text { DOG- } 1 \text { testing; } \mathbf{d} \text { Excluding } 31 \text { neoadjuvant patients who could not applicable to modified NIH criteria, } 70 \text { patients } \\
\text { left; e Only } 47 \text { patients underwent genetic testing. }\end{array}$} \\
\hline
\end{tabular}

\section{Analysis of patients receiving direct surgery and neoadjuvant therapy:}

Direct surgery: Seventy patients with the tumor size of $0.8-19.3 \mathrm{~cm}(5.63 \pm 3.28 \mathrm{~cm})$ received direct surgery, which included 8 very low risk, 10 low risk, 7 intermediate risk, and 45 high risk patients. Twenty-nine patients recurred or metastasized, including very low risk 1 case, low risk 1 case, intermediate risk 2 cases and high risk 25 cases, time to recurrence was $30.7 \pm 20.6$ months (5-96 months); Twenty-four of them died because of disease progression, including intermediate risk 2 cases, high risk 22 cases, the time of death was $51.5 \pm 22.4$ months (6-108 months). Cox regression analysis was performed on gender, age, diameter, invasiveness, ECOG Performance Score, surgical type, surgical margin, surgical scope, mitotic index, risk grade, post-operative IM adjuvant therapy, the results showed risk grade $(P=0.029)$ and post-operative IM adjuvant therapy $(P=0.001)$ affected RFS; Meanwhile, risk grade $(P=0.019)$ and post-operative IM adjuvant therapy $(P=0.033)$ were related to $O S$, too. Kaplan-Meier survival analysis of 70 patients undergoing direct surgery suggested that both risk classification and post-operative adjuvant therapy affected the RFS and OS, Kaplan-Meier of risk classification were showed in Fig. 1.

Excluding 18 patients with very low risk and low risk patients, 52 intermediate and high risk patients left, Cox regression showed that post-operative IM adjuvant treatment improved their DFS $(P=0.003)$ and OS $(P=0.027)$. Kaplan-Meier of post-operative IM adjuvant treatment were showed in Fig. 2.

IM neoadjuvant therapy: Thirty-one patients received IM neoadjuvant therapy for 3-15 months (6.1 \pm 2.5 months) : complete response (CR) 0, partial response (PR) 26, stable disease (SD) 4 and progressive disease (PD) 1.The objective response rate(ORR)was $83.9 \%$ (26/31), and the disease control rate (DCR) reached $96.8 \%$ (30/31). The diameter before treatment was $3.60-11.90 \mathrm{~cm}(7.23 \pm 1.90 \mathrm{~cm})$, and the neoadjuvant therapy lasted $3-15$ months $(6.1 \pm 2.5$ months). After treatment, the diameter was $1.70-9.50 \mathrm{~cm}(4.77 \pm 1.67 \mathrm{~cm})$, but the difference was not statistically significant $(P=0.465)$. The images of some patients before and after IM neoadjuvant therapy are shown in Fig. 3. Durning the follow-up, six patients recurred, time to recurrence was $49.5 \pm 16.9$ months (26-69 months), and 4 of them died, the time of death was $59.5 \pm 19.7$ months (33-78 months).

\section{Subgroup analysis of diameter}


(1) Among 36 patients with a tumor diameter $\leq 5 \mathrm{~cm}$, two patients received IM neoadjuvant therapy, and 34 patients received direct surgery. No factors (gender, age, ECOG Performance Score,invasion, type of surgery, surgical margin, scope of surgery, scope of surgery, mototic index, IM neoadjuvant therapy and IM adjuvant therapy) affecting RFS and OS were found in univariate analysis and Cox regression analysis.

(2) Among the 65 patients with a tumor diameter $>5 \mathrm{~cm}, 29$ patients received IM neoadjuvant therapy and 36 patients received direct surgery. No significant difference was observed in operative time $(P=0.621)$ and recovery time of gastrointestinal function $(P=0.222)$ between the two groups. However, the neoadjuvant treatment group was superior to the direct surgery group in terms of blood loss $(P=0.022)$ and post-operative hospital stay $(P=0.001)$, the anal preservation rate increased $(P=0.031)$, and the stoma rate decreased $(P=0.037)$. Details are shown in Table 2. Kasquare univariate analysis indicated that invasiveness of the surrounding organs $(P<0.001)$, neoadjuvant therapy $(P$ $=0.002)$, Scope of surgery $(P=0.022)$, mitotic index $(P=0.001)$, and adjuvant treatment of $I M$ after surgery $(P<0.001)$ affected DFS, while gender $(P=0.918)$, age $(P=0.437)$, ECOG Performance Score $(P=0.367)$, type of surgery $(P=$ $0.741)$ and surgical margin $(P=0.133)$ did not affect DFS. Among the above ten factors, Cox regression analysis indicated invasiveness of the surrounding organs $(P=0.040)$, neoadjuvant treatment $(P=0.028)$ and adjuvant imatinib $(P=0.003)$ affected DFS, the other seven factors had no effect. Meanwhile, none of the above ten factors were found affectting OS. Kaplan-Meier survival analysis suggested that invasion of the surrounding organs $(\mathrm{P}<$ $0.001)$, neoadjuvant therapy $(P<0.001)$ and post-operative IM adjuvant therapy $(P<0.001)$ affected recurrence and metastasis, as shown in the Fig. 4. Remove deleted patients, the 3-year DFS, 3-year OS, 5-year DFS, 5-year OS of the patients who received IM neoadjuvant therapy and those who received direct surgery were $(23 / 25) 92 \%$ VS (18/36) $50 \%, P=0.001 ;(24 / 25) 92 \%$ VS $(32 / 36) 88.9 \%, P=0.602 ;(13 / 16) 81.3 \%$ VS $(10 / 32) 31.3 \%, P=0.001 ;(13 / 16) 81.3 \%$ VS $(16 / 32) 50 \%, P=0.007$.

Table 2

Comparison of surgical conditions of 65 patients with diameter $>5 \mathrm{~cm}$

\begin{tabular}{|llllllll|}
\hline Group & Number & $\begin{array}{l}\text { Operation } \\
\text { time } \\
\text { (min) }\end{array}$ & $\begin{array}{l}\text { Bleeding } \\
\text { amount } \\
\text { (ml) }\end{array}$ & $\begin{array}{l}\text { Recovery time } \\
\text { of } \\
\text { gastrointestinal } \\
\text { function (day) }\end{array}$ & $\begin{array}{l}\text { Postoperative } \\
\text { hospital stay } \\
\text { (day) }\end{array}$ & $\begin{array}{l}\text { Anus reser- } \\
\text { vation } \\
\text { proportion }\end{array}$ & $\begin{array}{l}\text { Enterosto- } \\
\text { my } \\
\text { proportion }\end{array}$ \\
\hline $\begin{array}{l}\text { Neoadjuvant } \\
\text { group }\end{array}$ & 29 & $\begin{array}{l}120.0 \pm \\
35.5\end{array}$ & $\begin{array}{l}91.2 \pm \\
17.7\end{array}$ & $2.9 \pm 0.9$ & $7.3 \pm 2.3$ & $27 / 29$ & $3 / 29$ \\
$\begin{array}{l}\text { Direct } \\
\text { Surgery }\end{array}$ & 36 & $\begin{array}{l}154.6 \pm \\
38.0\end{array}$ & $22.3 \pm$ & $3.7 \pm 1.2$ & $10.3 \pm 4.9$ & $26 / 36$ & $12 / 36$ \\
\hline P value & & 22.3 & & & $(72.2 \%)$ & $(33.3 \%)$ \\
\hline
\end{tabular}

\section{Discussion}

Rectal GIST is relatively rare, accounting for only $5 \%$ of GISTs, our study showed rectal GISTs were $4.2 \%$ of all GISTs (138/3316), similar to the Rebecca report [8]. The median size of rectal GISTs was $5 \mathrm{~cm}[9,10]$, but the median size obtained in our study was $6.18 \pm 3.02 \mathrm{~cm}$, which was slightly larger than that of the above studies. The clinical symptoms of rectal GISTs are related to the size of the tumor. When the tumor is less than $2 \mathrm{~cm}$, no clinical symptoms are observed, and they are often found in the physical examination occasionally. However, as the tumor gradually grows into the intestinal cavity, a series of symptoms occur, including stool trait change, defecation habits change, anal discomfort or tumor rupture caused by blood in stool, and late invasion of the surrounding organs can also be manifested as hemuria and vaginal bleeding [11]. Our study showed that rectal bleeding, change in bowel of stool, 
change in bowel habit and anal discomfort, were $19.8 \%, 14.9 \%, 17.8 \%$ and $16.8 \%$, respectively. It is worth mentioning that $17.8 \%$ of the patients did not have any symptoms, but were found initally durning the rectal palpation. Therefore, its clinical manifestations have no specific manifestations compared to other rectal diseases. However, rectal GISTs tends to occur in the lower segments $[9,10]$, in our group, $78.2 \%(79 / 101)$ patients had tumors located in the lower segments similar to most studies, and $69.3 \%$ (70/101) of them could be found by rectal palpation. Therefore, rectal palpation plays an important role in the early discovery and differential diagnosis of rectal GISTs.

The pathological diagnosis of rectal GISTs is mainly based on histological and immunohistochemical results [12]. The cell morphology was divided into spindle, epithelioid, and mixed cell type, which accounted for $97.0 \%, 96.2 \%$ and $82.1 \%$ in our study, respectively, similar to GISTs in other sites. In terms of immunohistochemistry, CD117 and DOG-1 had the most diagnostic value, and CD34 was very significant for its diagnosis. Miettinen et al. reported that the expression rates of CD117 and CD34 in 96 cases of rectal mesenchymal tumors were $100 \%$ and $94 \%$, respectively [13]. In our study, the positive rates of CD117 and DOG-1 were 97.0\% (98/101) and 96.2\%(75/78), and CD34 also reached $82.1 \%(83 / 101)$, thus, CD117 was similar to Miettinen's report, but CD34 was slightly lower. According to Miettinen' report, KIT exon 11 mutation are common in rectal GISTs, followed by exon 9 mutation and wild-type mutation, but PDGFRA mutations are rare in rectal GISTs. Similar results were obtained in the present study, where KIT exon 11 (38/47), KIT exon 9 (4/47) and wild-type (4/47) were detected, however, no PDGFRA mutation patientswere found in our study. Rectal GISTs often has a layer of pseudo-parcapsule on the surface, rarely infiltrate along the intestinal wall, and rarely has lymph node metastasis. Hence,lymph node dissection is not necessary [14]. Among the 101 patients in the present study,76 lymph nodes were dissected, but no metastasis occurred, thus confirming the above view.

Rectal GIST is a disease with high recurrence rate. Surgical resection is still the mostimportant treatment. The surgical principle is to complete excision, maintain the integrity of the capsule, and avoid rupture. The malignant risk of rectal GIST is higher than that ofstomach and is closer to that of intestinal GIST. Yasui, et al. reported that the proportion of rectal high risk GISTs was 45\%, while the MSKCC single center reported $72.3 \%[4,15]$. For the 70 patients that underwent direct surgery, 45 cases were at high risk after surgery, accounting for $64.3 \%$, which was higher than the result obtained by Yasui. However, considering that 31 patients with large diameter could not be evaluated by modified NIH criteria after IM neoadjuvant therapy, our ratio of high risk would be higher than this value, supporting the data of MSKCC. Cox regression analysis was performed on gender, age, diameter, invasiveness, ECOG Performance Score, surgical type, surgical margin, surgical scope, mitotic index, risk classification, post-operative IM adjuvant therapy of seventy patients, the results showed risk grade and post-operative IM adjuvant therapy affected DFS and OS. Therefore, for patients with intermediate and high risk rectal GISTs, postoperative adjuvant treatment with imatinib is particularly important for improving their prognosis.

For patients with large tumors, prone to intra-operative bleeding, and tumors close to the anal margin, IM neoadjuvant therapy can be considered, and this treatment will result in obvious tumor descent effect, improve the anal preservation rate, reduce the positive rate of surgical margin' and improve the prognosis of patients [16-19]. At present, the time of IM neoadjuvant therapy is appropriate within 6-12 months according to NCCN and ESMO guidelines $[16,17]$. Each guideline recommends that the duration of neoadjuvant treatment be defined as the maximum response to medication. The maximum response time was defied as two consecutive enhanced CT or MRI scans indicate no remission of the tumor. At this time, surgical resection should be performed immediately given an opportunity for surgery [20]. With prolonged drug treatment time, secondary mutations may occur during treatment. Bednarski et al., in a retrospective study of 93 patients treated pre-operatively, showed that the neoadjuvant treatment time of $>365$ days was associated with an increased progression rate [21]. Therefore, blindly prolonging the IM neoadjuvant treatment time to maximize is highly likely to lead to drug resistance and then miss the best operative 
timing [22]. In our present study, 31 patients were treated for 3-15 months (6.1 \pm 2.5 months). During IM neoadjuvant treatment, the patients were generally followed up and evaluated dynamically every 3 months timely to understand the effect of neoadjuvant therapy and accurately determine the timing of surgery. In the present study, the ORR was $83.9 \%$, and the DCR reached as high as $96.8 \%$. This result was similar to the Kanedo report involving 6 retrospective studies in 118 patients with neoadjuvant rectal stromal tumors with a response rate of $70.3 \%$ and a control rate of $99.2 \%[23]$.

Considering that the rectal GIST is located in the narrow pelvis with special anatomical structure and adjacent to important structures such as the reproductive and urinary systems, diameter of the tumor is the most important indicator for us to consider IM neoadjuvant therapy. Thus, we conducted a subgroup analysis of the diameter, and found that no factors, including IM neoadjuvant therapy, affecting DFS and OS in patients with a diameter of $\leq 5 \mathrm{~cm}$. But, considering the small sample size, whether IM neoadjuvant therapy in patients with a diameter of $\leq 5 \mathrm{~cm}$ can provide survival benefit for rectal GISTs needs to be further verified. However, for patients with tumor diameter $>5 \mathrm{~cm}$, univariate analysis $(P=0.002)$ and multivariate analysis $(P=0.028)$ both indicated neoadjuvant therapycan improve DFS. So, it was the same as Vallilas's report that IM neoadjuvant therapy for specific sites or large tumors can improve the prognosis [15]. Meanwhile, it is worth mentioning that in our study, whether the surgical margin was positive or not did not affect the prognosis and there was no need for further surgical resection, which was consistent with Cavnar's and Gronchi's report [24,25]. In summary, for patients with a diameter $\leq 5 \mathrm{~cm}$, considering that the composition of IM neoadjuvant therapy was relatively low, with only 2 cases, the data analysis might be biased. Whether neoadjuvant therapy in patients with a diameter $\leq 5 \mathrm{~cm}$ can provide survival benefit for rectal GISTs needs to be further verified in a multi-center, large-sample prospective study. However, for rectal GISTs with diameter $>5 \mathrm{~cm}$, our univariate and Cox regression analysis both showed that neoadjuvant treatment could improve the prognosis of patients. It could improve 3-year RFS and 5-year RFS and OS. Therefore, for rectal GIST patients with a diameter of $>5$ $\mathrm{cm}$, we recommend IM neoadjuvant therapy and then receive operation in order to tumor's descending and improve the prognosis.

Moreover, from subgroup analysis of the diameter, our research showed that the neoadjuvant therapy of diameter > $5 \mathrm{~cm}$ could improve safety of the surgery, preserve the anus, decrease possibilities of enterostomy and improve the post-operative quality of life of patients. At present, how to shrink the tumors that are difficult to be completely resected and how to improve the anal preservation rate of patients with low rectal GISTs have been widely focused on [26]. It has been reported IM neoadjuvant therapy can reduce bleeding and improve safety of the surgery, which may be attributed to the reduction of tumor volume and the fibrosis, hyaline degeneration, and toughening of tumor texture caused by drugs, making it less prone to rupture and bleeding during surgery. Our study also confirmed the value of neoadjuvant therapy with shorter postoperative hospital stay $(P=0.001)$ and less bleeding $(P=0.022)$. Moreover, neoadjuvant therapy can significantly reduce the tumor diameter, and this condition is conducive to the implementation of organ preservation surgery $[27,28]$. The MSKCC single-center study showed that IM neoadjuvant adjuvant therapy significantly increased the rate of anal preservation ( $92 \%$ vs. $48 \%$ ). Our study showed that neoadjuvant therapy could increase the anal retention rate $(93.1 \%$ vs. $72.2 \%, P=0.031)$ and reduce the rate of enterostomy $(10.3 \%$ vs. $33.3 \%, P=0.037)$, demonstrating the value of IM neoadjuvant therapy in preserving organ function.

In conclusion, rectal GIST is a disease with special location, high malignancy and recurrence rate. Post-operative IM adjuvant treatment can reduce the recurrence and metastasis rate of the intermediate and high risk patients. IM neoadjuvant therapy can reduce tumor volume, protect organ structure and function, and improve prognosis of patients with a diameter $>5 \mathrm{~cm}$. However, the neoadjuvant treatment of rectal GISTs remains lacking standard at 
domestic and abroad. In the future, more prospective multi-center studies are needed to further explore and draw conclusions.

\section{Abbreviations}

GISTs: Gastrointestinal stromal tumors; IM: Imatinib mesylate; DFS: Disease-free survival (DFS); OS: Overall survival; $\mathrm{NIH}$ : National Institutes of Health; CR: complete response; PR: partial response; SD:stable disease; PD: progressive disease; ORR: Objective response rate; DCR: disease control rate; ECOG: Eastern Cooperative Oncology Group.

\section{Declarations}

\section{Acknowledgements}

No support was received from any organization for the submitted work. The authors have no financial relationships to disclose.

\section{Authors' contributions}

$B Z$ and QW designed the study. HXY, CYS, XNY and ZLC contributed to analysis and interpretation of the date. HXY and CYS were major contributors in writing the manuscript. All authors read and approved the final manuscript.

\section{Funding information}

This work was supported by the Project Sichuan Science and Technology Support Program (Grant No. 2020YFS0234) and 1.3.5 project for disciplines of excellence, West China Hospital, Sichuan University (ZYJC18034).

\section{Availability of data and materials}

The datasets used and analyzed during the current study are available from the corresponding author on reasonable request.

\section{Ethics approval and consent to participate}

All methods were carried out in accordance with relevant guidelines and regulations. The study protocol, conform to the ethical guidelines of the 1975 Declaration of Helsinki. And, this study was approved by the Ethics Committee of the West China Hospital of Sichuan University (Chengdu, China) and the Affiliated Hospital of Guizhou Medical University (Guiyang, China). Written informed consents were obtained from all the participants.

\section{Consent for publication}

Wirtten informed consent was obtained from all individual participants included in the study.

\section{Competing interests}

None of the authors has a potential conflict of interest with respect to the research, authorship and publication of this article.

\section{References}


1. Parab TM, Derogatis MJ, Boaz AM, et al. Gastrointestinal stromal tumors: a comprehensive review. J Gastrointest Oncol. 2019;10(1):144-54. https://doi.org/10.21037/jgo.2018.08.20.

2. Soreide K, Sandvik OM, Soreide JA, et al. Global epidemiology of gastrointestinal stromal tumours (GIST): a systematic review of population-based cohort studies. Cancer Epidemiol.2016;40:39-46. https://doi.org/10.1016/j.canep.2015.10.031.

3. Mohamad F, Johnny $\mathrm{O}$, Claramae $\mathrm{C}$, et al. Treatment of gastrointestinal tumor (GIST) of the rectum requiring abdominoperineal resection following neoadjuvant imatinib:a cost-effectiveness analysis. Clin Sarcoma Res.2020;10(13):1-11. https://doi.org/10.1186/s13569-020-00135-7.

4. Yasui $\mathrm{M}$, Tsujinaka $\mathrm{T}$, Mori $\mathrm{M}$, et al. Characteristics and prognosis of of rectal gastrointestinal stromal tumors: an analysis of registry data. Surg Today.2017;47(10):1188-94. https://doi.org/10.1007/s00595-017-1524-8.

5. Chen L, Hao W, Han L,et al. Different Medical Features and Strategies of Large Rectal Gastrointestinal Stromal Tumor: A Multi-Central Pooling Analysis. Cancer Management and Research.2021;13:1591600. https://doi.org/10.2147/CMAR.S291269.

6. Joensuu H. Risk stratification of patients diagnosed with gastrointestinal stromal tumor. Hum Pathol. 2008;39:1411-9. https://doi.org/10.1016/j.humpath.2008.06.025.

7. Choi H囚Response evaluation of gastrointestinal stromal tumors $₫$ Oncologist.2008,13(Suppl 2):4-7. https://doi.org/10.1634/theoncologist.13-S2-4.

8. Rebecca Z, Fangfang L, Gabriella G, et al. Distinctive features of gastrointestinal stromal tumors arising from the colon and rectum. J Gastrointest Oncol.2018;9(2):231-40. https://doi.org/10.21037/jgo.2017.11.12.

9. Hawkins A, Wells K, Krishnamurty D, et al. Preoperative Chemotherapy and Survival for Large Anorectal Gastrointestinal Stromal Tumors: A National Analysis of 333 Cases. Ann Surg Oncol.2017;24(5): 1195-201. https://doi.org/10.1245/s10434-016-5706-1.

10. Cavnar M, Wang L, Balachandran V, et al. Rectal Gastrointestinal Stromal Tumor (GIST) in the era of imatinib: Organ preservation and improved oncologic outcome. Ann Surg Oncol.2017;24(13):3972-80. https://doi.org/10.1245/s10434-017-6087-9.

11. Li J, Ye Y, Wang J, et al. Chinese consensus guidelines for diagnosis and management of gastrointestinal stromal tumor. Chin J Cancer Res.2017;29(4):281-93. https://doi.org/10.21147/j.issn.1000-9604.2017.04.01.

12. Kane WJ, Friel CM. Diagnosis and Treatment of Rectal Gastrointestinal Stromal Tumors. Dis Colon Rectum.2019;62(5):537-40. https://doi.org/10.1097/DCR.0000000000001376.

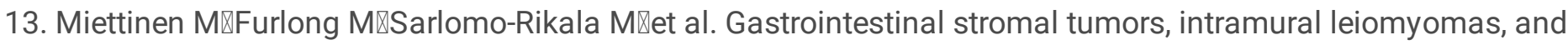
leiomyosarcomas in the rectum and anus-A clinicopathologic, immunohistochemical, and molecular genetic study of 144 cases. Am J Surg Pathol.2001;25(9):1121-33. https://doi.org/10.1097/00000478-20010900000002.

14. Von MM『Randall RL『Benjamin RS『et al. Soft tissue sarcoma, version 2, 2016, NCCN clinical practice guidelines in oncology. J Natl Compr Canc Netw.2016;14(6):758-86. https://doi.org/10.6004/jnccn.2016.0078.

15. Vallilas C, Sarantis P, Kyriazoglou A, et al. Gastrointestinal Stromal Tumors (GISTs): Novel therapeutic strategies with immunotherapy and small molecule. Int J Mol Sci.2021: 22(2):E493.

https://doi.org/10.3390/ijms22020493.

16. Von MM, Randall RL, Benjamin RS, et al. Soft Tissue Sarcoma, Version 2.2018, NCCN Clinical Practice Guidelines in Oncology.Journal of the National Comprehensive Cancer Network: JNCCN,2018, 16(5): 536-563. https://doi.org/10.6004/jnccn.2018.0025. 
17. Casali PG, Abecassis N, Aro HT, et al. Gastrointestinal stromal tumours: ESMO-EURACAN Clinical Practice Guidelines for diagnosis, treatment and follow-up. Ann Oncology.2018;29(Suppl 4):iv267. https://doi.org/10.1093/annonc/mdy320.

18. Masaaki I, Kazuto H, Shiro I, et al. Neoadjuvant and adjuvant therapy for gastrointestinal stromal tumors. Annals Gastroenterol.2019;3(1);43-49. https://doi.org/10.1002/ags3.12211.

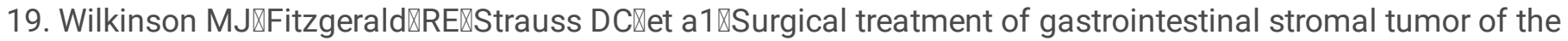
rectum in the era of imatinib $\otimes B r$ J Surg.2015;102(8)ष965-71. https://doi.org/10.1002/bjs.9818.

20. Wang SY, Wu CE, Lai CC, et al. Prospective evaluation of neoadjuvant Imatinib use in locally advanced gastrointestinal stromal tumors:emphasis on the optimal duration of neoadjuvant Imatinib use, safety, and oncological outcome. Cancers.2019;11(424):1-12. https://doi.org/10.3390/cancers11030424.

21. Bednarski BK\Araujo DM $₫$ Yi M\et al. Analysis of prognostic factors impacting oncologic outcomes after neoadjuvant tyrosine kinase inhibitor therapy for gastrointestinal stromal tumors. Ann Surg Oncol.2014;21(8):2499₫505. https://doi.org/10.1245/s10434-014-3632-7.

22. Yong ZZ, Wong JSM, Teo MCC, et al.Neoadjuvant tyrosine kinase inhibitors in rectal gastrointestinal stromal tumours: a provision for enhanced oncological and functional outcomes. Int J Clin Oncol.2021;26(5):913-21. https://doi.org/10.1007/s10147-021-01867-2.

23. Kaneko M, Emoto S, Murono K, et al. Neoadjuvant imatinib therapy in rectal gastrointestinal stromal tumors. Surg Today.2019;49(6):460-6. https://doi.org/10.1007/s00595-018-1737-5.

24. Cavnar MJ, Seier K, Curtin C, et al. Outcome of 1000 patients with gastrointestinal stromal tumor (GIST) treated by surgery in the pre- and post-imatinib eras. Ann Surg.2021,273(1):1-21. https://doi.org/10.1097/SLA.0000000000003277.

25. Gronchi A, Bonvalot S, Velasco AP, et al. Quality of Surgery and Outcome in Localized Gastrointestinal Stromal Tumors Treated Within an International Intergroup Randomized Clinical Trial of Adjuvant Imatinib.JAMA Surg.2020,155(6):e200397. https://doi.org/10.1001/jamasurg.2020.0397.

26. Aneko M, Nozawa $\mathrm{H}$, Emoto S, et a1 $₫$ Neoadjuvant imatinib therapy followed by intersphincteric resection for low rectal gastrointestinal stromal tumors $₫$ Anticancer Res.2017;37(9):5155-60》 https://doi.org/10.21873/anticanres.11936.

27. Tielen $\mathrm{R}$, Verhoef $\mathrm{C}$, van Coevorden $\mathrm{F}$, et al. Surgical management of rectal gastrointestinal stromal tumors. J Surg Oncol.2013;107(4):320-3. https://doi.org/10.1002/jso.23223.

28. Jakob J, Mussi C, Ronellenfitsch U, et al. Gastrointestinal stromal tumor of the rectum: results of surgical and multimodality therapy in the era of imatinib. Ann Surg Oncol.2013;20(2):586-92. https://doi.org/10.1245/s10434012-2647-1.

\section{Figures}



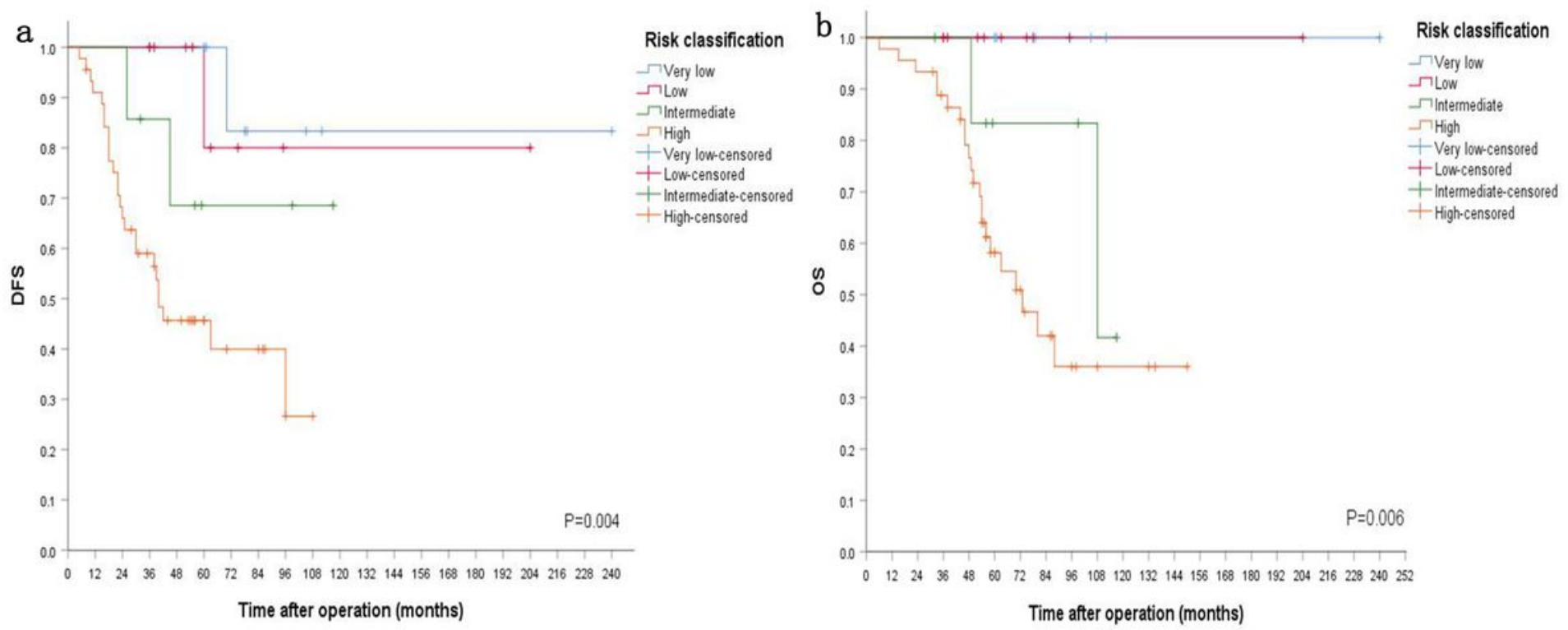

\section{Figure 1}

Prognosis of of 70 patients who received direct surgery. a DFS stratified by modified NIH risk classification. b OS stratified by modified NIH risk classification.
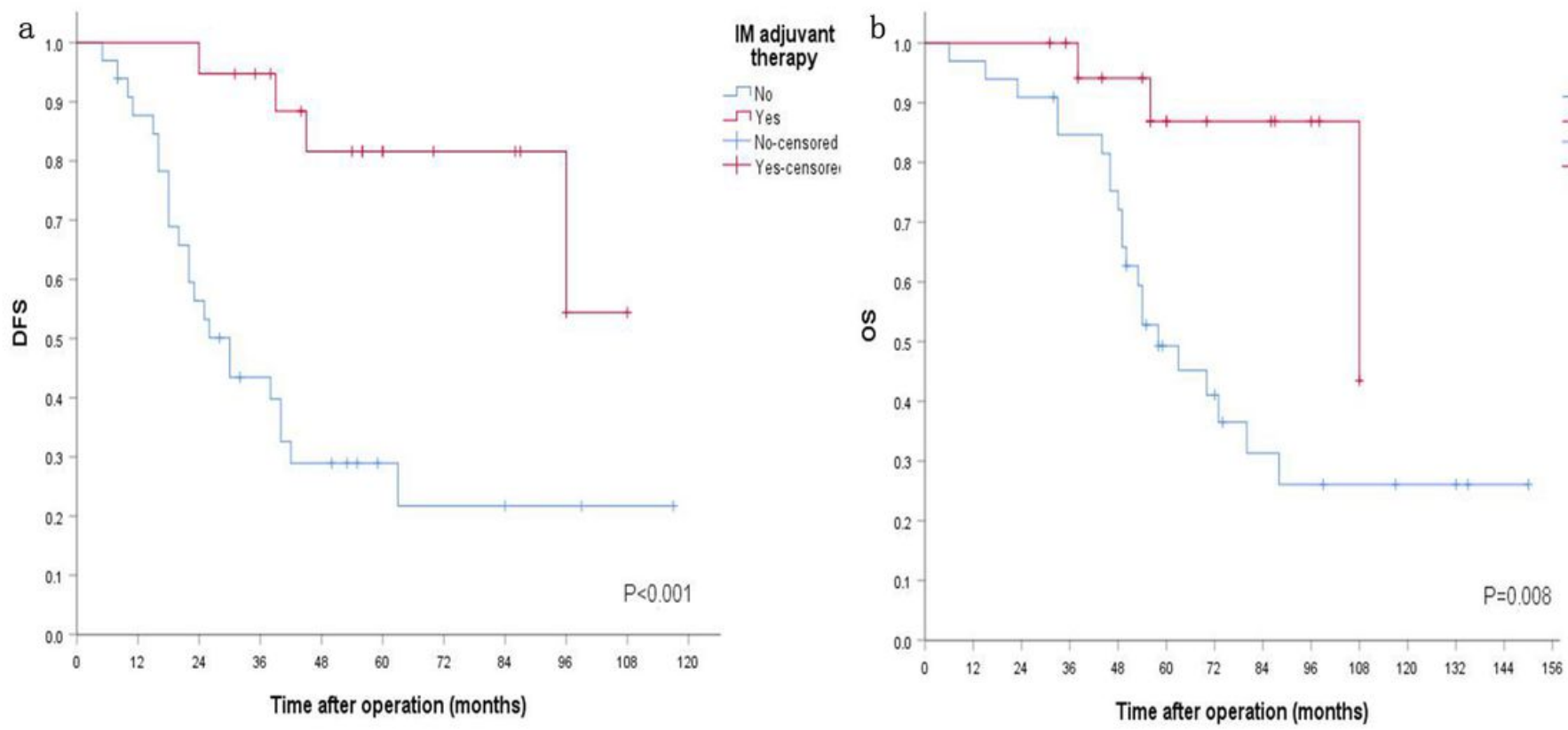

\section{Figure 2}

Prognosis of of 52 patients with intermediate and high risk receiving direct surgery. a DFS stratified by post-operative IM adjuvant treatment. b OS stratified by post-operative IM adjuvant treatment. 

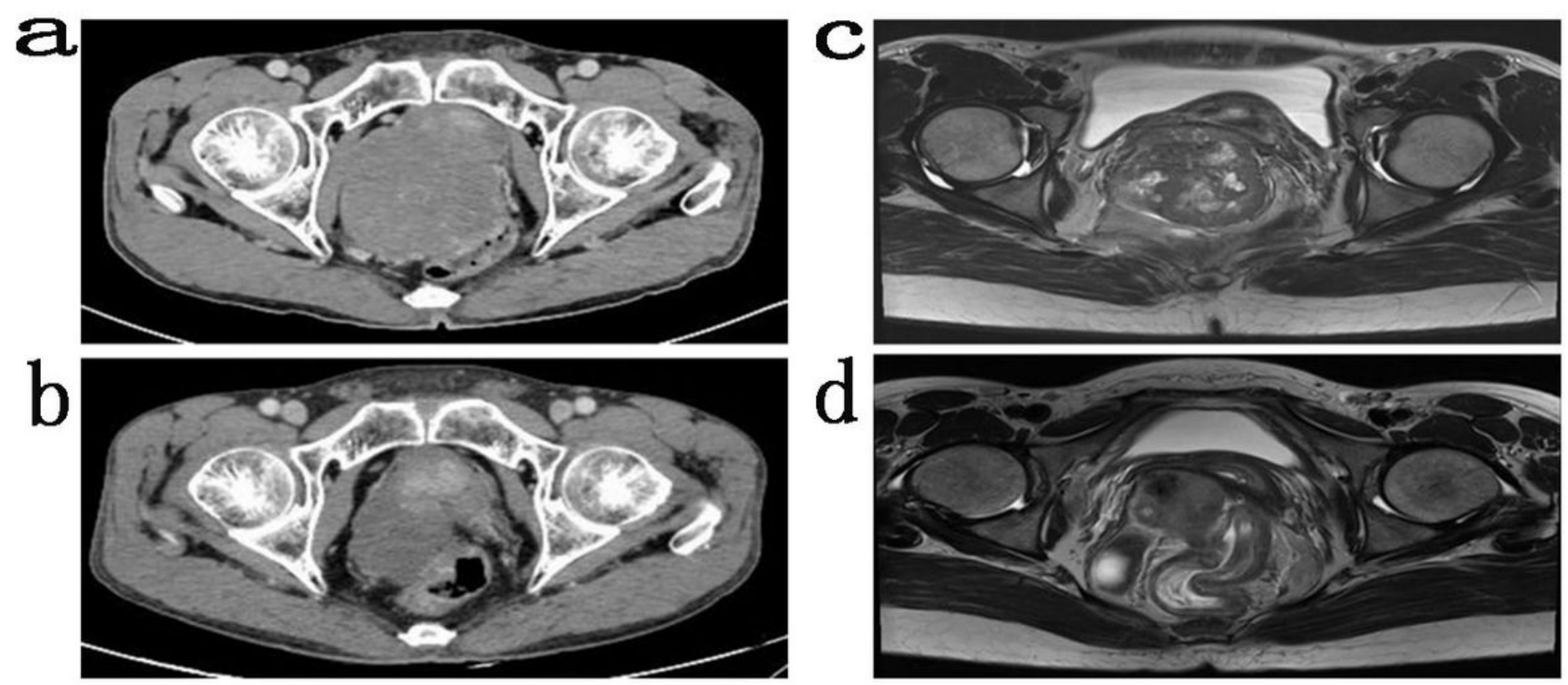

\section{Figure 3}

Imaging comparison of two patients before and after IM neoadjuvant treatment. Enhanced CT scan of the rectal GIST patient received IM neoadjuvant therapy for 6 months (a initial tumor, and b tumor after 6 months of IM neoadjuvant therapy). Magnetic resonance imaging of another rectal GIST patient treated with IM neoadjuvant therapy for 9 months (c initial tumor, and d tumor after 9 months IM neoadjuvant therapy) 


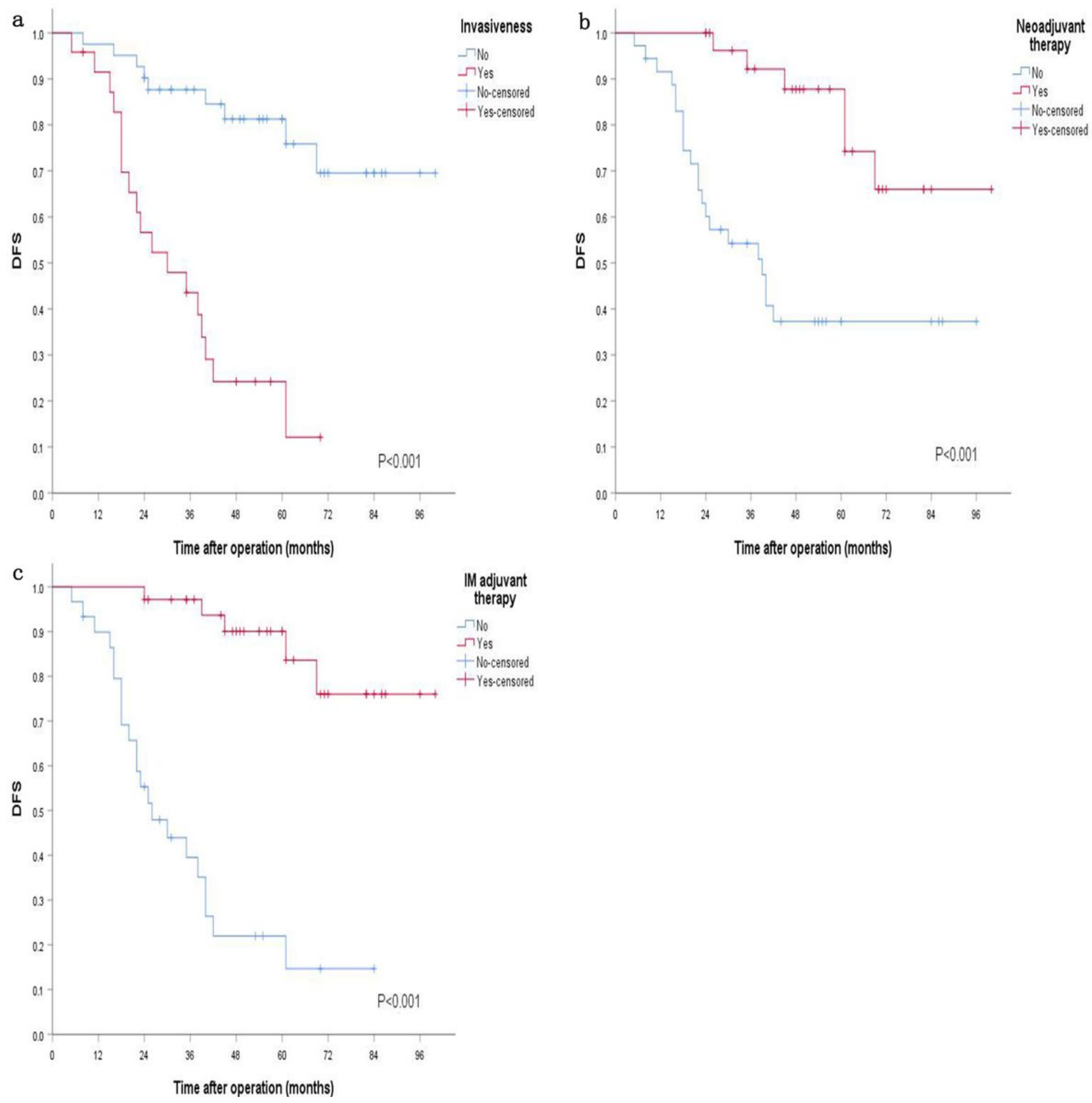

Figure 4

DFS of patient with diameter $>5 \mathrm{~cm}$ stratified by invasiveness, neoadjuvant and post-operative treatment. a DFS stratified by invasiveness. b DFS stratified by neoadjuvant IM treatment. c DFS stratified by post-operative IM adjuvant treatment. 